\title{
Optimization of Machining Parameters for GFRP Composite in CNC Lathe Using Anova
}

\author{
M Mohan \\ Assistant Professor, Department of mechanical Engineering \\ Ganesh College of Engineering - 636 111, Tamil Nadu. India. \\ A Balamurugan \\ Assistant Professor, Department of mechanical Engineering \\ Ganesh College of Engineering - 636 111, Tamil Nadu. India. \\ M Ramakrishnan \\ Assistant Professor, Department of mechanical Engineering \\ Ganesh College of Engineering - 636 111, Tamil Nadu. India. \\ A Gowrishankar \\ Assistant Professor, Department of mechanical Engineering \\ Ganesh College of Engineering - 636 111, Tamil Nadu. India. \\ V Jagadeeshwar \\ Assistant Professor, Department of mechanical Engineering \\ Ganesh College of Engineering - 636 111, Tamil Nadu. India.
}

\begin{abstract}
Nowadays, glass fiber reinforced plastics (GFRP) composites play a vital role in many engineering applications as an alternative to various heavy exotic materials. A GFRP polymeric composite, the matrix of polymer (resin) is reinforced with glass line fibers. Glass fiber reinforced plastics are increasingly used for variety of engineering applications from automobile to air craft components because of their superior advantages when compared to the other engineering materials. The advantages include weight-to-strength ratio, high fracture toughness, excellent thermal and corrosion resistance. Though the technology of composite manufacturing is advanced, near-net-shaped components with the required surface finish quality can be achieved only by machining. Surface quality and dimensional precision will greatly affect the parts during their useful life, especially in cases where the components will be in contact with other elements or materials during their useful life. Therefore, their study and characterization is extremely important. There are significant differences between the machining of metals, alloys and that of composite materials, because composites are an isotropic and inhomogeneous in nature. Yet no special machines have been developed to machine composite materials, still traditional metal cutting tools and techniques are being used. It was appropriate to study the behavior when machining GFRP composite with different types of tools and optimization of process parameters (Ex: cutting speed, feed, and depth of cut and fiber orientation angle) influencing machinability to achieve high productivity with low cost manufacturing. Hence, in the present research work, an attempt has been made to investigate the machining characteristics of GFRP composite tubes of different fiber orientation with various process parameter was carried out to the surface roughness (Ra), Machining time to be analyzed. The analysis of means (ANOM) was performed to determine the optimal levels of the parameters and the analysis of variance (ANOVA) was employed to identify the level of importance of the machining parameters on Surface roughness (Ra) and machining time.
\end{abstract}

Keywords: Optimization, Machining Parameters, GFRP, Anova

\section{INTRODUCTION}

\subsection{GLASS FIBRE REINFORCED PLASTICS}

Over $95 \%$ of the fibers used in reinforced plastics are glass fibers, as they are inexpensive, easy to manufacture and possess high strength and stiffness with respect to the plastics, with which they are reinforced. 
Their low densities, resistance to chemicals, insulation capacity are the other bonus characteristics of the glass fiber. Glass fibers are available in the form of mats, tapes, cloths, continuous and chopped filaments, roving and yarns. Addition of chemicals to silica sand while making glass yields different types of glasses. The main types are E-glass (also called "fiberglass") and S-glass. The E in E-glass stands for electrical as it was designed for electrical applications as an insulator. However, it is used for many other purposes now such as decorations and structural applications. The S in S-glass stands for higher content of silica. It retains its strength at high temperature as compared to E-glass and has higher fatigue strength. It is used mainly for aerospace applications. Other type of fibers available are C-glass (C stands for corrosion) used in chemical environments such as storage tanks, R-glass used in structural application such as constructions. D glass (D stands for dielectric) used for applications requiring low dielectric constants such as radomes, and A-glass (A stands for appearance) used to improve surface appearance.

\subsection{ANALYSIS OF VARIANCE}

The present contribution of various process parameters on the selected characteristics can be estimated by performing ANOVA. Thus the information about how significant effect of each controlled process parameter is obtained. This section of the help is intended to provide you with definitions for each statistic in the analysis of variance. Some statistics are only given under certain conditions or for certain designs. The ANOVA is built entirely on the premise that the factors are fixed, not random and the design is crossed, not nested. Note that we can choose View, Annotated ANOVA to provide assistance with interpretation of the results.

\subsection{INPUT PARAMETERS}

\section{EXPERIMENTAL PROCEDURE}

\begin{tabular}{|c|c|c|c|}
\hline Sample & Speed, rpm & Feed, mm/rev & DOC, $\mathrm{mm}$ \\
\hline 1 & 1200 & 0.03 & 0.2 \\
\hline 2 & 1200 & 0.05 & 0.4 \\
\hline 3 & 1200 & 0.07 & 0.6 \\
\hline 4 & 1700 & 0.03 & 0.4 \\
\hline 5 & 1700 & 0.05 & 0.6 \\
\hline 6 & 1700 & 0.07 & 0.2 \\
\hline 7 & 2200 & 0.03 & 0.6 \\
\hline 8 & 2200 & 0.05 & 0.2 \\
\hline 9 & 2200 & 0.07 & 0.4 \\
\hline
\end{tabular}

Table 2.1 Input Parameters 


\begin{tabular}{|c|c|c|c|c|c|c|}
\hline $\begin{array}{l}\text { Trial } \\
\text { No. }\end{array}$ & Designation & Speed, rpm & Feed, mm/rev & DOC, mm & $\begin{array}{c}\text { Ra in } \\
\text { microns }\end{array}$ & $\begin{array}{c}\text { Machining } \\
\text { Time in mins. }\end{array}$ \\
\hline 1 & $\mathrm{~A}_{1} \mathrm{~B}_{1} \mathrm{C}_{1}$ & 1200 & 0.03 & 0.2 & 2.494 & 106 \\
\hline 2 & $\mathrm{~A}_{1} \mathrm{~B}_{2} \mathrm{C}_{2}$ & 1200 & 0.05 & 0.4 & 2.757 & 60 \\
\hline 3 & $\mathrm{~A}_{1} \mathrm{~B}_{3} \mathrm{C}_{3}$ & 1200 & 0.07 & 0.6 & 2.698 & 44 \\
\hline 4 & $\mathrm{~A}_{2} \mathrm{~B}_{1} \mathrm{C}_{2}$ & 1700 & 0.03 & 0.4 & 2.296 & 120 \\
\hline 5 & $\mathrm{~A}_{2} \mathrm{~B}_{2} \mathrm{C}_{3}$ & 1700 & 0.05 & 0.6 & 2.347 & 43 \\
\hline 6 & $\mathrm{~A}_{2} \mathrm{~B}_{3} \mathrm{C}_{1}$ & 1700 & 0.07 & 0.2 & 2.629 & 33 \\
\hline 7 & $\mathrm{~A}_{3} \mathrm{~B}_{1} \mathrm{C}_{3}$ & 2200 & 0.03 & 0.6 & 2.876 & 53 \\
\hline 8 & $\mathrm{~A}_{3} \mathrm{~B}_{2} \mathrm{C}_{1}$ & 2200 & 0.05 & 0.2 & 3.696 & 34 \\
\hline 9 & $\mathrm{~A}_{3} \mathrm{~B}_{3} \mathrm{C}_{2}$ & 2200 & 0.07 & 0.4 & 2.295 & 27 \\
\hline
\end{tabular}

After finding all the observation as given in table 2.1, $\mathrm{S} / \mathrm{N}$ ratio and means are calculated and also various graph for analysis is drawn by using Minitab-15 software. The $\mathrm{S} / \mathrm{N}$ ratio for Ra, Machining time and MRR is calculated on Minitab -15 Software using Taugchi Method.

\section{SURFACE ROUGHNESS (ANALYSIS OF RESULT)}

\subsection{SURFACE ROUGHNESS AND S/N RATIOS VALUES}

\begin{tabular}{|c|c|c|c|c|c|c|}
\hline $\begin{array}{l}\text { Trial } \\
\text { No. }\end{array}$ & Designation & Speed, rpm & Feed, mm/rev & DOC, mm & $\begin{array}{c}\text { Ra in } \\
\text { microns }\end{array}$ & $\begin{array}{c}\text { S/N Response } \\
\text { valve (db) for } \\
\text { Ra }\end{array}$ \\
\hline 1 & $\mathrm{~A}_{1} \mathrm{~B}_{1} \mathrm{C}_{1}$ & 1200 & 0.03 & 0.2 & 2.494 & -7.93793 \\
\hline 2 & $\mathrm{~A}_{1} \mathrm{~B}_{2} \mathrm{C}_{2}$ & 1200 & 0.05 & 0.4 & 2.757 & -8.80874 \\
\hline 3 & $\mathrm{~A}_{1} \mathrm{~B}_{3} \mathrm{C}_{3}$ & 1200 & 0.07 & 0.6 & 2.698 & -8.62084 \\
\hline 4 & $\mathrm{~A}_{2} \mathrm{~B}_{1} \mathrm{C}_{2}$ & 1700 & 0.03 & 0.4 & 2.296 & -7.21944 \\
\hline 5 & $\mathrm{~A}_{2} \mathrm{~B}_{2} \mathrm{C}_{3}$ & 1700 & 0.05 & 0.6 & 2.347 & -7.41026 \\
\hline 6 & $\mathrm{~A}_{2} \mathrm{~B}_{3} \mathrm{C}_{1}$ & 1700 & 0.07 & 0.2 & 2.629 & -8.39581 \\
\hline 7 & $\mathrm{~A}_{3} \mathrm{~B}_{1} \mathrm{C}_{3}$ & 2200 & 0.03 & 0.6 & 2.876 & -9.17578 \\
\hline 8 & $\mathrm{~A}_{3} \mathrm{~B}_{2} \mathrm{C}_{1}$ & 2200 & 0.05 & 0.2 & 3.696 & -11.3546 \\
\hline 9 & $\mathrm{~A}_{3} \mathrm{~B}_{3} \mathrm{C}_{2}$ & 2200 & 0.07 & 0.4 & 2.295 & -7.21565 \\
\hline
\end{tabular}

\subsection{ANALYSIS OF VARIANCE (ANOVA)}

Analysis of Variance (ANOVA) results for the Roughness

\begin{tabular}{|ccccccc|}
\hline $\begin{array}{c}\text { Source of } \\
\text { variation }\end{array}$ & DOF & $\begin{array}{c}\text { Sum of } \\
\text { squares(S) }\end{array}$ & Variance(v) & F ratio(F) & $\begin{array}{c}\text { P value } \\
\text { (p) }\end{array}$ & $\begin{array}{c}\text { \% of } \\
\text { contribution }\end{array}$ \\
speed & 2 & 0.21572 & 0.10786 & 10.24 & 0.027 & 80.50 \\
feed & 2 & 0.00498 & 0.00249 & 0.24 & 0.800 & 3.87 \\
ERROR & 4 & 0.04213 & 0.01053 & & & 15.70 \\
TOTAL & 9 & 0.26284 & & & & $\mathbf{1 0 0}$ \\
\hline
\end{tabular}

Response for Roughness

\begin{tabular}{|cccc|}
\hline Level & $\mathbf{A}$ & $\mathbf{B}$ & $\mathbf{C}$ \\
1 & 2.750 & $\mathbf{2 . 5 3 3}$ & 2.940 \\
2 & 2.424 & 2.933 & $\mathbf{2 . 4 5 0}$ \\
3 & $\mathbf{2 . 9 6 6}$ & 2.541 & 2.640 \\
Delta $\Delta_{\text {max-min }}$ & 0.542 & 0.400 & 0.490 \\
Rank & $\mathbf{1}$ & $\mathbf{3}$ & $\mathbf{2}$ \\
\hline
\end{tabular}

Volume 8 Issue 2 April 2017 

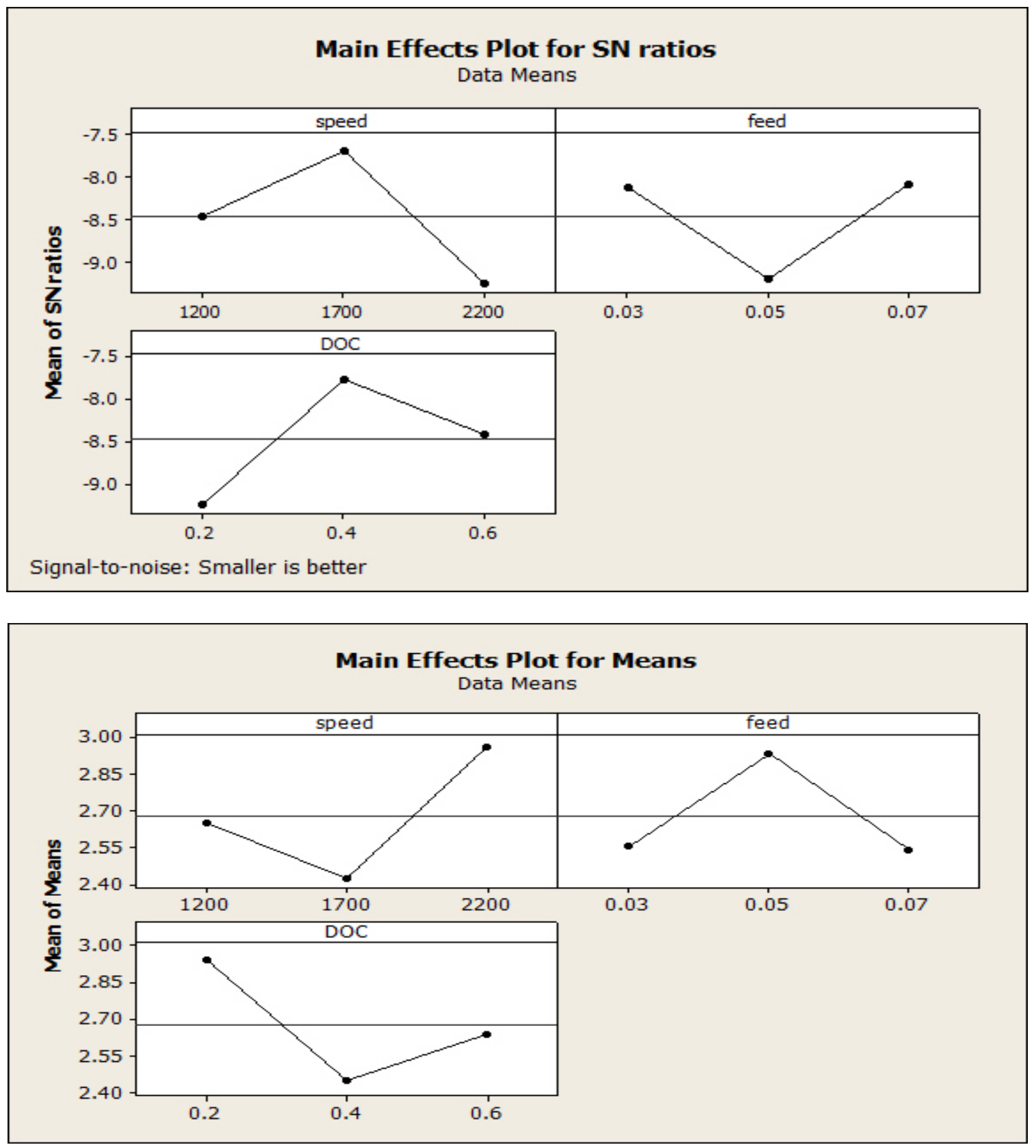

Optimal level values for Roughness from graph

\begin{tabular}{|cccc|}
\hline Process parameters & Levels & $\begin{array}{c}\text { Roughness response } \\
\text { value }\end{array}$ & $\begin{array}{c}\text { S/N response value } \\
(\mathbf{d b})\end{array}$ \\
A & 1 & 2.750 & -8.456 \\
B & 3 & 2.541 & -8.077 \\
C & 2 & 2.450 & -7.748 \\
\hline
\end{tabular}




\section{MACHINING TIME (ANALYSIS OF RESULT)}

4.1 MACHINING TIME AND S/N RATIOS VALUES

\begin{tabular}{|c|c|c|c|c|c|c|}
\hline $\begin{array}{l}\text { Trial } \\
\text { No. }\end{array}$ & Designation & Speed, rpm & Feed, mm/rev & DOC, mm & $\begin{array}{c}\text { Machining } \\
\text { Time in SEC }\end{array}$ & $\begin{array}{c}\text { S/N Response } \\
\text { valve (db) }\end{array}$ \\
\hline 1 & $\mathrm{~A}_{1} \mathrm{~B}_{1} \mathrm{C}_{1}$ & 1200 & 0.03 & 0.2 & 106 & -40.5061 \\
\hline 2 & $\mathrm{~A}_{1} \mathrm{~B}_{2} \mathrm{C}_{2}$ & 1200 & 0.05 & 0.4 & 60 & -35.5630 \\
\hline 3 & $\mathrm{~A}_{1} \mathrm{~B}_{3} \mathrm{C}_{3}$ & 1200 & 0.07 & 0.6 & 44 & -32.8691 \\
\hline 4 & $\mathrm{~A}_{2} \mathrm{~B}_{1} \mathrm{C}_{2}$ & 1700 & 0.03 & 0.4 & 120 & -41.5836 \\
\hline 5 & $\mathrm{~A}_{2} \mathrm{~B}_{2} \mathrm{C}_{3}$ & 1700 & 0.05 & 0.6 & 43 & -32.6694 \\
\hline 6 & $\mathrm{~A}_{2} \mathrm{~B}_{3} \mathrm{C}_{1}$ & 1700 & 0.07 & 0.2 & 33 & -30.3703 \\
\hline 7 & $\mathrm{~A}_{3} \mathrm{~B}_{1} \mathrm{C}_{3}$ & 2200 & 0.03 & 0.6 & 53 & -34.4855 \\
\hline 8 & $\mathrm{~A}_{3} \mathrm{~B}_{2} \mathrm{C}_{1}$ & 2200 & 0.05 & 0.2 & 34 & -30.6296 \\
\hline 9 & $\mathrm{~A}_{3} \mathrm{~B}_{3} \mathrm{C}_{2}$ & 2200 & 0.07 & 0.4 & 27 & -28.6273 \\
\hline
\end{tabular}

4.2 ANALYSIS OF VARIANCE (ANOVA)

Analysis of Variance (ANOVA) results for the MACHINING TIME

\begin{tabular}{|ccccccc|}
\hline $\begin{array}{c}\text { Source of } \\
\text { variation }\end{array}$ & DOF & $\begin{array}{c}\text { Sum of } \\
\text { squares(S) }\end{array}$ & Variance(v) & F ratio(F) & $\begin{array}{c}\text { P value } \\
\text { (p) }\end{array}$ & $\begin{array}{c}\text { \% of } \\
\text { contribution }\end{array}$ \\
speed & 2 & 1792.9 & 896.4 & 2.98 & 0.161 & 20.47 \\
feed & $\mathbf{2}$ & $\mathbf{5 7 6 4 . 2}$ & $\mathbf{2 8 8 2 . 1}$ & $\mathbf{9 . 5 9}$ & $\mathbf{0 . 0 3 0}$ & $\mathbf{6 5 . 8 0}$ \\
ERROR & 4 & 1202.4 & 300.6 & & & 13.73 \\
TOTAL & 9 & 8759.6 & & & & $\mathbf{1 0 0}$ \\
\hline
\end{tabular}

Response for MACHINING TIME

\begin{tabular}{|cccc|}
\hline Level & $\mathbf{A}$ & $\mathbf{B}$ & $\mathbf{C}$ \\
1 & 70 & $\mathbf{9 3}$ & 57.67 \\
2 & $\mathbf{6 5 . 3 3}$ & 45.67 & 69 \\
3 & 43 & 34.67 & $\mathbf{4 6 . 6 7}$ \\
Delta $\Delta_{\text {max-min }}$ & 4.67 & 58.33 & 11.33 \\
Rank & $\mathbf{2}$ & $\mathbf{1}$ & $\mathbf{3}$ \\
\hline
\end{tabular}



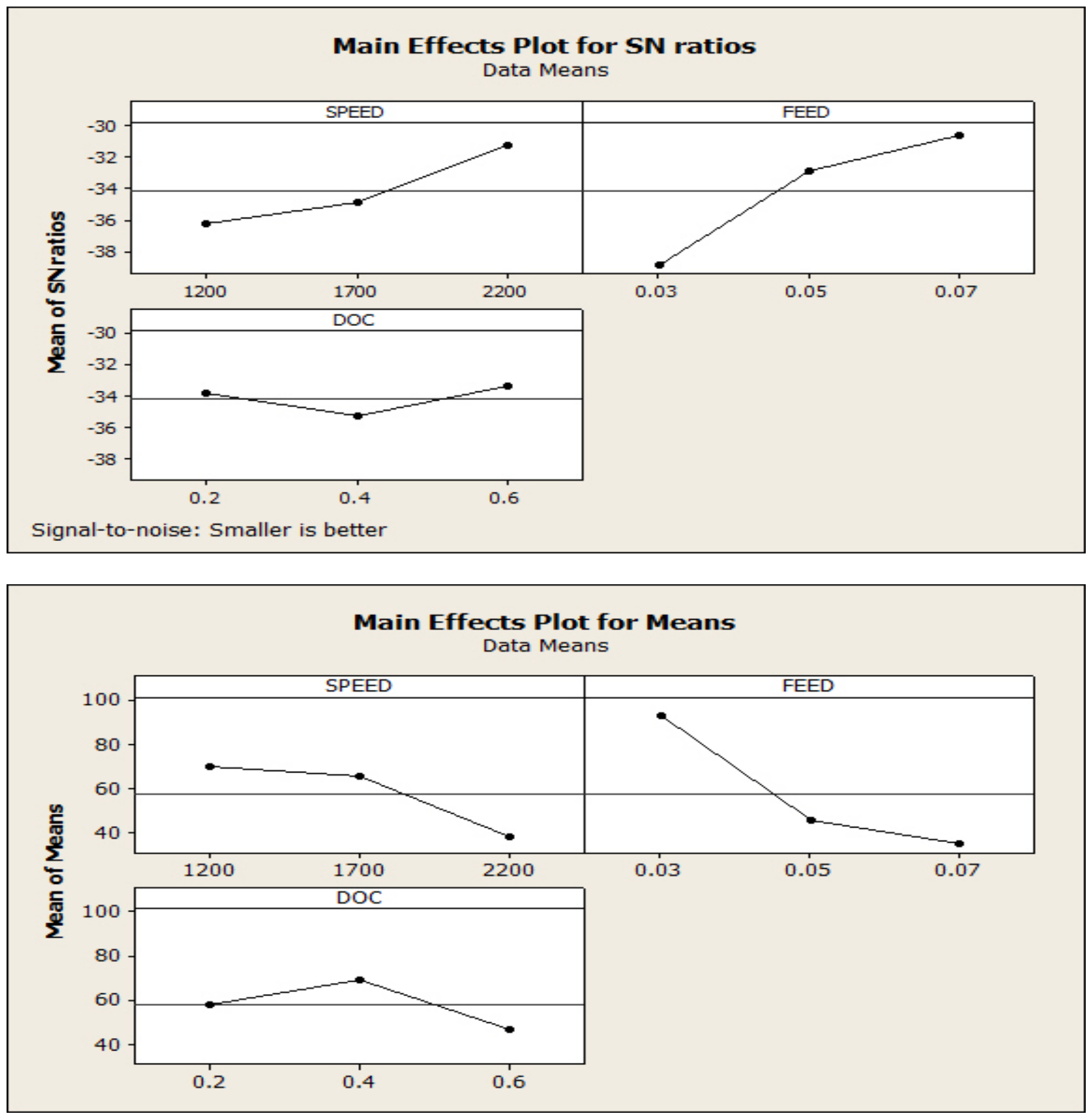

Optimal level values for MACHINING TIME from graph

\begin{tabular}{|cccc|}
\hline Process parameters & Levels & $\begin{array}{c}\text { MACHINING TIME } \\
\text { response value }\end{array}$ & $\begin{array}{c}\text { S/N response value } \\
(\mathbf{d b})\end{array}$ \\
A & 2 & 65.33 & -31.25 \\
B & 1 & 93 & -38.86 \\
C & 3 & 46.67 & -35.26 \\
\hline
\end{tabular}

V. MRR (ANALYSIS OF RESULT)

5.1 MRR AND S/N RATIOS VALUES

\begin{tabular}{|l|l|l|l|l|l|c|}
\hline $\begin{array}{l}\text { Trial } \\
\text { No. }\end{array}$ & Designation & Speed, rpm & Feed, $\mathbf{m m} / \mathbf{r e v}$ & DOC, $\mathbf{m m}$ & $\begin{array}{c}\text { MRR in } \\
\mathbf{m m}^{3} / \mathrm{sec}\end{array}$ & $\begin{array}{c}\text { S/N Response } \\
\text { valve (db) for } \\
\text { MRR }\end{array}$ \\
\hline
\end{tabular}

Volume 8 Issue 2 April 2017 


\begin{tabular}{|c|c|c|c|r|r|c|}
\hline 1 & $\mathrm{~A}_{1} \mathrm{~B}_{1} \mathrm{C}_{1}$ & 1200 & 0.03 & 0.2 & 1.179528 & 1.4342 \\
\hline 2 & $\mathrm{~A}_{1} \mathrm{~B}_{2} \mathrm{C}_{2}$ & 1200 & 0.05 & 0.4 & 2.118 & 6.5185 \\
\hline 3 & $\mathrm{~A}_{1} \mathrm{~B}_{3} \mathrm{C}_{3}$ & 1200 & 0.07 & 0.6 & 2.859545 & 9.1259 \\
\hline 4 & $\mathrm{~A}_{2} \mathrm{~B}_{1} \mathrm{C}_{2}$ & 1700 & 0.03 & 0.4 & 1.033167 & 0.2834 \\
\hline 5 & $\mathrm{~A}_{2} \mathrm{~B}_{2} \mathrm{C}_{3}$ & 1700 & 0.05 & 0.6 & 2.937442 & 9.3594 \\
\hline 6 & $\mathrm{~A}_{2} \mathrm{~B}_{3} \mathrm{C}_{1}$ & 1700 & 0.07 & 0.2 & 3.866061 & 11.7454 \\
\hline 7 & $\mathrm{~A}_{3} \mathrm{~B}_{1} \mathrm{C}_{3}$ & 2200 & 0.03 & 0.6 & 2.439245 & 7.7451 \\
\hline 8 & $\mathrm{~A}_{3} \mathrm{~B}_{2} \mathrm{C}_{1}$ & 2200 & 0.05 & 0.2 & 3.717647 & 11.4054 \\
\hline 9 & $\mathrm{~A}_{3} \mathrm{~B}_{3} \mathrm{C}_{2}$ & 2200 & 0.07 & 0.4 & 4.758519 & 13.5494 \\
\hline
\end{tabular}

5.2 ANALYSIS OF VARIANCE (ANOVA)

Analysis of Variance (ANOVA) results for the Roughness

\begin{tabular}{|c|c|r|r|r|r|c|}
\hline Source & DF & SS & MS & F & P & \% of contribution \\
\hline speed & 2 & 3.8824 & 1.9412 & 19.53 & 0.009 & 30.56 \\
\hline feed & 2 & 7.8903 & 3.9451 & 39.68 & 0.002 & 64.83 \\
\hline Error & 4 & 0.3976 & 0.0994 & & & 3.267 \\
\hline Total & 8 & 12.1703 & & & & 100 \\
\hline
\end{tabular}

Response for Roughness

\begin{tabular}{|cccc|}
\hline Level & A & B & C \\
1 & 2.750 & $\mathbf{2 . 5 3 3}$ & 2.940 \\
2 & 2.424 & 2.933 & $\mathbf{2 . 4 5 0}$ \\
3 & $\mathbf{2 . 9 6 6}$ & 2.541 & 2.640 \\
Delta $\Delta_{\text {max-min }}$ & 0.542 & 0.400 & 0.490 \\
Rank & $\mathbf{2}$ & $\mathbf{1}$ & $\mathbf{3}$ \\
\hline
\end{tabular}

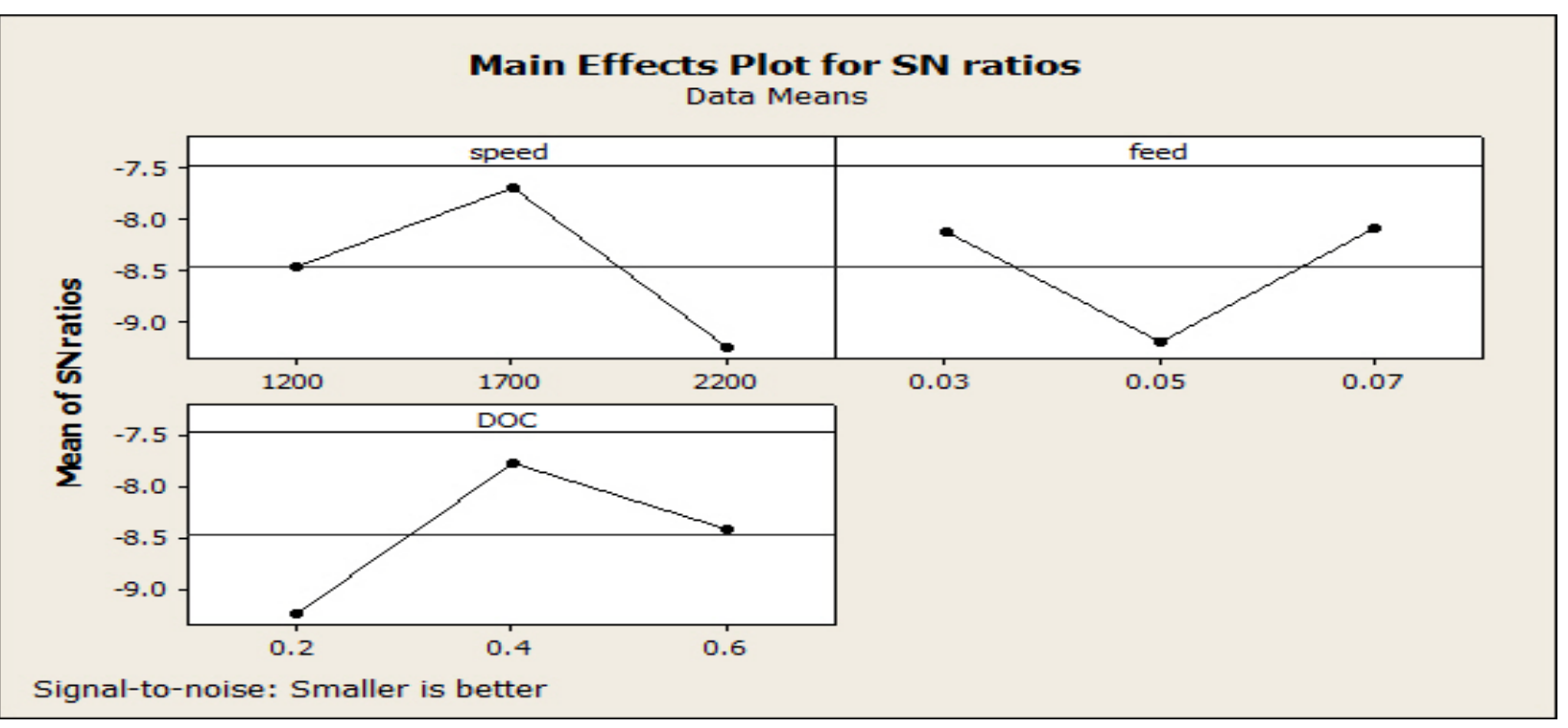




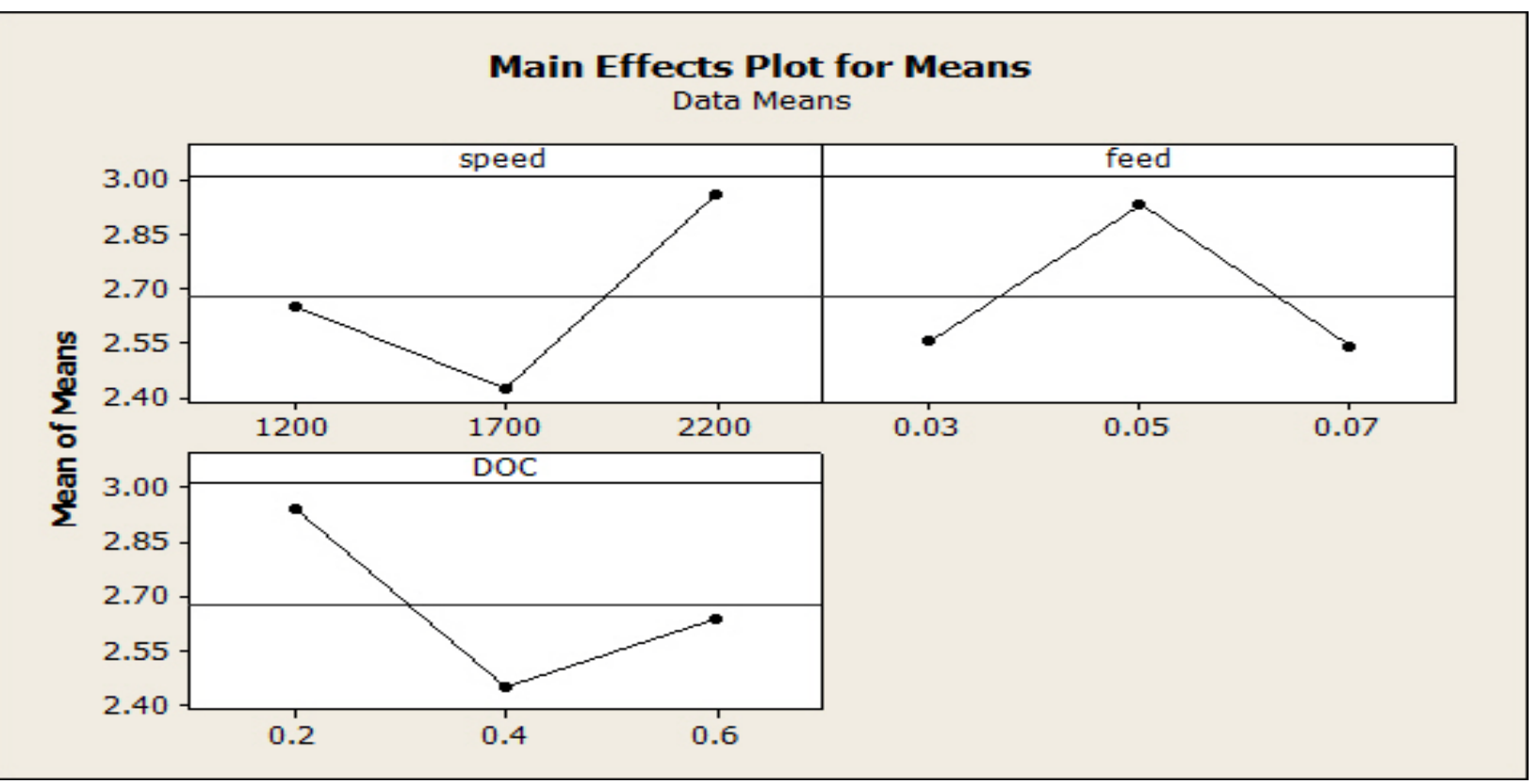

Optimal level values for Roughness from graph

\begin{tabular}{|cccc|}
\hline Process parameters & Levels & $\begin{array}{c}\text { Roughness response } \\
\text { value }\end{array}$ & $\begin{array}{c}\text { S/N response value } \\
\text { (db) }\end{array}$ \\
A & 2 & 2.414 & 7.129 \\
B & 1 & 2.555 & 3.154 \\
C & 3 & 2.640 & 8.743 \\
\hline
\end{tabular}

VI. CONCLUSION

In this study, the Taugchi technique and ANOVA were used to obtain optimal Turning parameters in the Turning of GFRP under dry conditions. The experimental results were evaluated using ANOVA. The following conclusion can be made.

Optimal Control Factor

1.Surface Roughness-A1(Speed -1200)B3(Feed -0.07)C2(DOC-0.4)

2.Machining Timing-A2(Speed-1700)B1(Feed 0.03)C3(DOC0.6)

3.Material Removal Rate- A2(Speed-1700)B1(Feed 0.03)C3(DOC0.6)

Percentage of Contribution of Process Parameter

1.Surface Roughness - speed $80 \%$

2.Machining Timing - Feed 66\%

3.Material Removal Rate- Feed 64\%

\section{REFERENCES}

[1] Syed AltafHussain, V. Pandurangadu, K. Palani Kumar "Cutting power prediction model for turning of GFRP composites using response surface methodology” International Journal of Engineering, Science and Technology Vol. 3, No. 6, 2011, pp. 161-171

[2] Syed AltafHussain, V. Pandurangadu, K. Palani Kumar "Machinability of glass fiber reinforced plastic(GFRP) composite material" International Journal of Engineering, Science and Technology Vol. 3, No. 4, 2011, pp. 103-118.

[3] S.Aravindan, A.NaveenSait, A.NoorulHaq, "Influence of machining parameters on surface roughness of GFRP pipes" advances in production \& management 4 (2009) 1-2, 47-58 SSN 1854-6250.

[4] S.Aravindan, A.NaveenSait, A.NoorulHaq, "optimization of machining parameters of GFRP pipes by desirability function analysis using taguchi technique ”Int J AdvManufTechnology (20o9),43: 581-589 .

[5] S.Aravindan, A.NaveenSait, A.NoorulHaq, "A Machinability Study of GFRP Pipes using Statistical Technique” Int J AdvManuf Technology (2008),37: 1069-1081.

[6] Reddy Sreenivasulu "Optimization of Surface Roughness and DelaminationDamage ofGFRP Composite Material in End Milling using Taguchi DesignMethod and Artificial Neural Network” International Conference onDESIGN AND MANUFACTURING, IConDM 2013.

[7] HariVasudevan,Naresh D Deshpande,RameshR.Rajguru "Desirability FuzzyMultiple Criteria Optimization of Process Parameter in CNC Turning of GFRP/Vinyl ester Composites, International Conference on Advances in Manufacturingand Materials Engineering,AMME 201. 